\title{
Research on Innovation Education Evaluation for Vocational College Based on Improved BP Neural Network
}

\author{
Hui Zhang \\ Electronic and Electrical Department, Wuhan Railway Vocational College of Technology, Wuhan, \\ 430205, China \\ 407706483@qq.com
}

\begin{abstract}
Keywords: Education performance management, Innovation education performance evaluation, BP
\end{abstract} neural network, Fourier basis functions.

\begin{abstract}
Along with the smooth advancement of a new round basic education reform, the profound changes have occurred in vocational college education ideology and teaching modes, which demands the reformation of innovation course education evaluation system to guarantee the acceleration of the reform. The paper presents a new model for evaluating innovation education for vocational college performance based on improved BP neural network. First an evaluation indicator system of innovation education performance is designed through the aspects of vocational college regulation and teachers and students and education effects; Second considering that BP neural network algorithm has high evaluation accuracy but low convergence, the paper adopts Fourier basis functions to improve traditional BPNN algorithm to speed up model convergence and to simplify model structure; Finally the experimental results show that the new evaluation indicator system and improved BP neural network algorithm can be used practically in evaluating innovation education for different vocational colleges and guarantee the evaluation effectiveness and validity.
\end{abstract}

\section{Introduction}

With the advent of the era of knowledge economy, national competitiveness is more and more reflected in the national innovation quality and innovation ability. Then more and more governments are committed to create "public entrepreneurship, people's innovation" as their economy engine, which can improve the quality of economic, increase economic efficiency and upgrade economic strength. So more and more vocational colleges try their best to deep innovation and entrepreneurship education reform and take it as their urgent necessities for national implementation of innovation driven development strategy. At the same time it is an important initiatives to promote comprehensive reform for vocational colleges to improve college graduates higher quality employment and entrepreneurship. As the main body of vocation education, science and engineering colleges undertake the important task of training the social elite talents, science and technology innovation talents and engineering technology applied talents. It is the basic bridge which linking modern technology and practical production requirements. Therefore the research of science and engineering specialties of vocational colleges of innovation and entrepreneurship education has important theoretical and practical significance to improve the college students innovation ability, alleviate the science and engineering student employment pressure and deepening teaching reform in vocational college[1,2].

\section{Literature Review}

Up to now, mathematical models adopted by evaluation of innovation education performance mainly include the following categories. (1) Analytic hierarchy process is a good method for quantitative evaluation via quantitative method, having the functions of establishing the ideal weight structure of evaluated object value and analyzing the weight structure of actually-built value by evaluated object; however, the method has strong limitations and subjectivity, with large personal error, not suitable for complicated system with lots of evaluation indicators[5]. (2) Fuzzy 
comprehensive evaluation is a method carrying out comprehensive evaluation and decision on system through fuzzy set theory, the greatest advantage of which is that it works well on system evaluation of multi-factor and multi-level complicated problems. However, the membership of fuzzy evaluation method as well as the definition and calculation of membership function are too absolute, difficult to reflect the dynamics and intermediate transitivity of evaluation indicators of innovation education performance[6,7]; (3)BP neural network evaluation method makes use of its strong capability in processing nonlinear problems to carry out evaluation of innovation education performance; the method has advantages like self-learning, strong fault tolerance and adaptability; however, the algorithm is easy to be trapped into defects like local minimum, over-learning, strong operation specialization[2,3]

The paper improves BP neural network by Fourier basis functions. In so doing, not only the problem of convergence speed of BPNN has been solved, but also the simplicity of the model structure and the accuracy of the evaluation are ensured.

\section{Analysis and Establishment of Evaluation Indicator System}

While designing the indicator system of innovation education evaluation of higher institutions, this thesis first takes the innovation education of higher institutions as characteristic. Higher education being a teaching activity transferring advanced knowledge and training senior professional talents, innovation education job in higher institutions, besides having the common characteristics of higher education job and common rules to be obeyed, has features different from ordinary education process. Therefore, this thesis first refers to literatures related to innovation education and experts' opinions, according to relevant principles of education and surveying, deciding the scope of influence of innovation education in higher institutions by combining area method with goal method, and designing evaluation indicator system with such four perspectives of innovation education job as schools, teachers, students and effects. The system includes 4 first-grade indicators, that is vocational colleges, teachers, students, education effects, in which first-grade indicator vocational colleges includes six second-grade indicators that are system construction, implementation organizing, funds and personnel input, construction of innovation base, education concept, innovation culture atmosphere in campus, totally 35 third-grade indicators.

\section{Derivation of Evaluation Algorithm}

\subsection{Continuous-Time Fourier Series of Periodic Signal}

As we all know, for signal $f(t)$ that the period is $T$, it can be showed by continuous-time Fourier series, i.e. Formula 1[7].

$$
\begin{gathered}
\mathrm{c} 2=\mathrm{a} 2+\mathrm{b} 2 . \\
f(t)=a_{0}+\sum_{n=1}^{\infty} a_{n} \cos \left(n \omega_{0} t\right)+\sum_{n=1}^{\infty} b_{n} \sin \left(n \omega_{0} t\right)
\end{gathered}
$$

Of Formula 2, $\omega_{0}=\frac{2 \pi}{T}$ is fundamental angular frequency, $a_{0}$ is DC component, and $a_{n} 、 b_{n}$ are Fourier series, i.e. Formula 2.

$$
a_{0}=\frac{1}{T} \int_{0}^{T} f(t) d t \quad a_{n}=\frac{2}{T} \int_{0}^{T} f(t) \cos \left(n \omega_{0} t\right) d t \quad b_{n}=\frac{2}{T} \int_{0}^{T} f(t) \sin \left(n \omega_{0} t\right) d t
$$

For time-limited nonperiodic signal $f(t), 0 \leq t \leq T$, the periodic signal that $f(t)$ is via continuation of period $T$ is $f_{p}(t)$, i.e. Formula 3[8].

$$
f_{p}(t)=\sum_{m=-\infty}^{\infty} f(t-m T)
$$


Of Formula 2, $m$ is a positive number. $f_{p}(t)=f(t)$ occurs obviously when time $t$ is $0 \leq t \leq T$. Therefore, the continuous-time series of periodic signal $f_{p}(t)$ can be also showed by Formula 1 within the principal value period $0 \leq t \leq T$.

For bandlimited signal $f(t)(0 \leq \omega \leq N \omega)$, Formula 1 can be changed as Formula 4[8].

$$
f(t)=a_{0}+\sum_{n=1}^{N} a_{n} \cos \left(n \omega_{0} t\right)+\sum_{n=1}^{N} b_{n} \sin \left(n \omega_{0} t\right)
$$

For the numerical computation, Formula 4 is separated into Formula 5.

$$
f(k)=a_{0}+\sum_{n=1}^{N} a_{n} \cos \left(n \omega_{0} k T_{s}\right)+\sum_{n=1}^{N} b_{n} \sin \left(n \omega_{0} t k T_{s}\right)
$$

Of Formula 6, $T_{s}$ is a sampling period, and $T_{s} \leq \frac{\pi}{N \omega_{0}}=\frac{T}{2 N}$. When $T_{s}=\frac{T}{2 N}$, Formula 5 can be changed as Formula 6.

$$
f(k)=a_{0}+\sum_{n=1}^{N} a_{n} \cos \left(\frac{\pi}{N} n k\right)+\sum_{n=1}^{N} b_{n} \sin \left(\frac{\pi}{N} n k\right)
$$

$k=0,1,2 \ldots . .2 N-1$ in Formula 6

\subsection{Neural Network Model Based on Fourier Basis Function}

In Formula 6, neural network model based on Fourier basis function is p roduced if $f(k)$ is a neural network output, $f_{d}(t)$ is a neural network training sample, $a_{n} 、 b_{n}$ are neural network training weights, and $\cos \left(\frac{\pi}{N} n k\right)$ and $\sin \left(\frac{\pi}{N} n k\right)$ are neural network excitation functions. See Fig. 1.

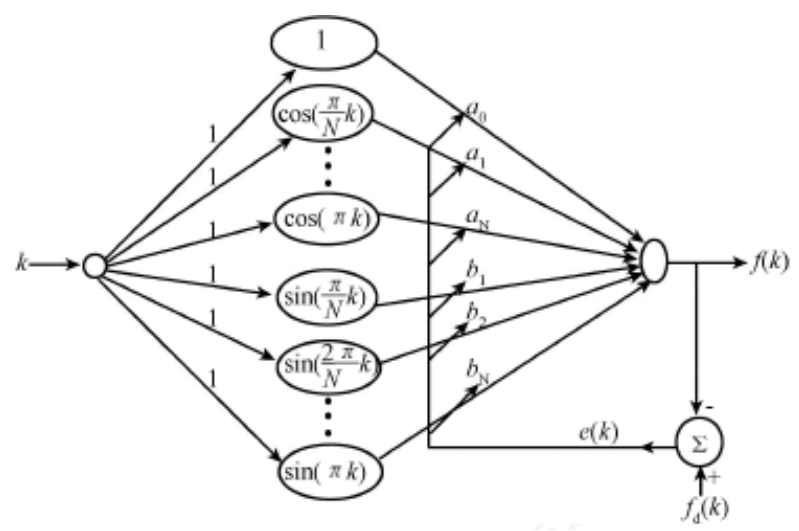

Fig. 1 Neural network model based on Fourier basis function

The algorithm of neural network model based on Fourier is as follows:

1. See Formula 6 for neural network output.

2. See Formula 7 for error function of network model.

$$
e(k)-f_{d}(k)-f(k)
$$

3. See Formula 9 for network model performance index.

4. Weight adjustment by gradient descent algorithm, See Formula 8 and 9 for weight adjustment quantity.

$$
\begin{aligned}
& \Delta a_{n}^{k}=-\eta \frac{\partial J}{\partial a_{n}^{k}}=\eta e(k) \cos \left(\frac{\pi}{N} n k\right), \quad n=0,1,2 \ldots N \\
& \Delta b_{n}^{k}=-\eta \frac{\partial J}{\partial b_{n}^{k}}=\eta e(k) \sin \left(\frac{\pi}{N} n k\right), \quad n=0,1,2 \ldots N
\end{aligned}
$$

5.See Formula 10 and 11 for weight adjustment, in which, $\eta$ is a learning rate, and $0 \prec \eta \prec 1$.

$$
a_{n}^{k+1}=a_{n}^{k}+\eta e(k) \cos \left(\frac{\pi}{N} n k\right), \quad n=0,1,2 \ldots N
$$




\section{Experiment Confirmation}

Experimental data come from database of Wuhan Railway Vocational College of Technology, and Wuhan Polytechnic and Shanghai Hubei Communication Technical College. Relevant data of 600 learner of each college are selected as the basis for data training and experimental verification in the paper, totally 1800 learns' data for study data that come from practical investigation and visit of different students. In order to make the selected learners' data representatives, 2400 learners(80 learner from each college) with more than 3 years learning experience, 1200 learners with 2 years learning experience, 360 learners with less than 2 years but more than one years learning experience. Limited to paper space, the evaluation of intermediate results is omitted here, only providing evaluation results of first grade indicators and final comprehensive evaluation results in table 1 and evaluation results of second grade indicators taking the first grade indicator of schools for example in table 2. from the evaluation results we know the presented algorithm and the evaluation indicator system are practical when used in innovation evaluation for vocational college.

Table 1 Evaluation results of first grade indicators

\begin{tabular}{lccc}
\hline & $\begin{array}{c}\text { Wuhan Railway Vocational } \\
\text { College of Technology }\end{array}$ & $\begin{array}{c}\text { Wuhan } \\
\text { Polytechnic }\end{array}$ & $\begin{array}{c}\text { Shanghai Hubei Communication } \\
\text { Technical College }\end{array}$ \\
\hline Schools & 4.135 & 4.127 & 4.087 \\
Teachers & 4.621 & 3.651 & 4.541 \\
Students & 4.421 & 3.541 & 4.387 \\
Education Effects & 4.321 & 3.431 & 4.211 \\
Final evaluation & 4.471 & 3.612 & 4.319 \\
\hline
\end{tabular}

Table 2 Part evaluation results of second grade indicators

\begin{tabular}{lccc}
\hline & $\begin{array}{c}\text { Wuhan Railway Vocational } \\
\text { College of Technology }\end{array}$ & $\begin{array}{c}\text { Wuhan } \\
\text { Polytechnic }\end{array}$ & $\begin{array}{c}\text { Shanghai Hubei Communication } \\
\text { Technical College }\end{array}$ \\
\hline $\begin{array}{l}\text { System Construction } \\
\text { Implementation }\end{array}$ & 4.218 & 3.891 & 4.192 \\
Organizing & 4.401 & 3.841 & 4.216 \\
$\begin{array}{l}\text { Funds and Personnel } \\
\text { Input }\end{array}$ & 4.402 & 3.761 & 4.264 \\
$\begin{array}{l}\text { Construction of } \\
\text { Innovation Base }\end{array}$ & 4.173 & 4.099 & 4.097 \\
$\begin{array}{l}\text { Education Concept } \\
\text { Innovation Culture }\end{array}$ & 4.312 & 4.100 & 4.201 \\
Atmosphere in Campus & 4.398 & 4.103 & 4.289
\end{tabular}

\section{References}

[1]. Zeng Haijun, Zeng Dekao. Strategy of Sustainable Development in Project of Modern Innovation Education in University. Computer Engineering and Design. vol 29(2015) No.3, p.659-661.

[2]. Zeng Haijun, Zeng Dekao. Designing E-Learning Environments for Activity and Instruction. Educational Technology. Research and Development. vol 52(2016).No.15, p.234-241.

[3]. John A Grete, Michel Green. Improving Undergraduate Learning with Computer Assisted Assessment. Journal of Research on Computering in Education.vol 35(2016) No.2, p.21-31.

[4]. J Wang Chunlian, Ma Xiufeng. Study on Progress Assessment System of Distance Education Using Clustering Analysis. Open Education Research. vol 12(2017) No.2, p.46-49.

[5]. Li Baoping. Design on the Evaluation System For Web-based Education. China Education. vol 12(2011) No.9, p.47-53. 
[6]. Yang Benzhao, Tian Gen. Research on Customer Value Classification based on BP Neural Network Algorithm. Science and Technology Management Research. vol. 23(2017) No.12, p.168-170.

[7]. Yi Su. Global Optimization for NN Training. IEEE Computers .vol. 19(2016) No.2, p. 45-54.

[8]. Xinmei Guo. The Study and Implementation of Back Propagation Neural Network Technology on Medical Statistics. JDCTA: International Journal of Digital Content Technology and its Applications. Vol. 7(2013) No. 2, p. 694-702. 